\title{
Noncontact scanning impedance imaging in an aqueous solution
}

Aaron R. Hawkins

hawkins@ee.byu.edu

Hongze Liu

Travis E. Oliphant

Stephen M. Schultz

Follow this and additional works at: https://scholarsarchive.byu.edu/facpub

Part of the Electrical and Computer Engineering Commons

\section{Original Publication Citation}

Hawkins, Aaron R., Hongze Liu, Travis E. Oliphant, and Stephen M. Schultz. "Noncontact scanning impedance imaging in an aqueous solution." Applied Physics Letters 85 (24): 18-182

\section{BYU ScholarsArchive Citation}

Hawkins, Aaron R.; Liu, Hongze; Oliphant, Travis E.; and Schultz, Stephen M., "Noncontact scanning impedance imaging in an aqueous solution" (2004). Faculty Publications. 422.

https://scholarsarchive.byu.edu/facpub/422 


\title{
Noncontact scanning impedance imaging in an aqueous solution
}

\author{
Aaron R. Hawkins, ${ }^{\text {a) }}$ Hongze Liu, Travis E. Oliphant, and Stephen M. Schultz \\ Electrical and Computer Engineering Department, Brigham Young University, 459 Clyde Building, \\ Provo, Utah 84602
}

(Received 3 March 2004; accepted 8 June 2004)

\begin{abstract}
We present a method for imaging based on noncontact electrical impedance measurements and mechanical scanning. Measurement results are shown for an initial system based on this concept. An impedance probe design is presented, applicable to the test system. Line-scan data plots of high impedance contrast structures show a good fit to a theoretical physical model. Image resolutions on the order of $100 \mu \mathrm{m}$ are indicated for the initial system. Two-dimensional impedance images of biological tissue generated by this technique are shown. (C) 2004 American Institute of Physics.
\end{abstract} [DOI: $10.1063 / 1.1778469]$

The imaging of a material's electrical impedance provides information that cannot be achieved with standard high-resolution surface profile techniques such as atomic force microscopy (AFM), scanning electron microscopy, near fields scanning optical microscopy, etc. Electrical impedance imaging has been previously achieved using tomographic reconstruction. These electrical impedance images can show useful information about human tissue composition ${ }^{1}$ allowing several medical applications including cancer diagnosis. ${ }^{2,3}$ However, the resolution of electrical impedance tomography is very low, limiting its application. The utility of electrical impedance imaging is also demonstrated by recent research that has incorporated electrical impedance measurements with a modified AFM in a direct contact configuration $^{4}$ and the single point measurements of blood cells in a liquid channel. ${ }^{5}$ The method of noncontact scanning impedance imaging (SII) described in this letter includes the resolution and imaging capabilities of scanning but eliminates the need to account for the complexity of contact resistance. This is especially important for soft tissues or materials with large impedances where contact resistance can vary significantly with surface conditions of both the probe and the material. Since SII can be done in a conducting aqueous solution, biological tissues can also be imaged in vitro. A high-resolution electrical impedance image of biological tissues could reveal a great deal of information due to the biologically significant behavior of electrically active ions (e.g., sodium, potassium, chlorine, and calcium), polar molecules (e.g., water), and inhomogeneous charge distributions (e.g., most proteins).

The process of noncontact scanning impedance imaging requires three elements: (1) an impedance probe, (2) a conducting solution surrounding the material under test, and (3) a system for precise scanning over a test sample. Immersion of the probe in a conductive solution allows for innovative probe design without the constraint of making reliable electrical contacts directly between the probe and the material under test. Figure 1 illustrates the components of a SII system. As shown in the figure, the impedance of a sample is measured between the end of a probe and a conducting plane, with water of $10 \mathrm{k} \Omega \mathrm{cm}$ resistivity filling the space between the sample and the probe. This system is useful mainly for imaging relatively flat materials without large changes in their thickness. A Newmark Systems Model NLS4-4-16 XYZ stage controller was used to move the probe. For all test results shown in this letter, a $2.5 \mathrm{~V}$ peakto-peak, $1 \mathrm{kHz}$ ac signal was used to drive current though the probe with a $100 \mathrm{k} \Omega$ resistor $(R)$ used for conversion to a voltage signal.

Initial testing using the system illustrated in Fig. 1 was done using a Teflon ${ }^{\mathrm{TM}}$ insulator $2.75 \mathrm{~mm}$ wide and $0.77 \mathrm{~mm}$ high sitting on a copper plate. Probes of two basic designs were used. The first consisted simply of a metal wire embedded in an insulator. The end of the wire and insulator was polished exposing a "point source probe" with dimensions equal to the wire's area. The second type of probe used a coaxial geometry with a central wire surrounded by insulator and then a subsequent conductor. The idea behind this "shielded probe" design was to eliminate the unwanted current flux toward the central wire from areas far from the probe. This concept is illustrated in Fig. 2 along with a crosssectional diagram of the shielded probe. When used in the prototype system in Fig. 1 the resistor $R$ ensures that the outside conductor (shield) is at a higher voltage potential than the central wire (tip). This ensures that current flux to the tip can come only from an area beneath the probe of total diameter equal to the tip diameter $(D)$ plus twice the shield spacing $\left(S_{p}\right)$.

Impedance measurements were drastically different depending on the type and size of the probe used. Figure 3(a) shows some of the results for line-scans over the Teflon in-

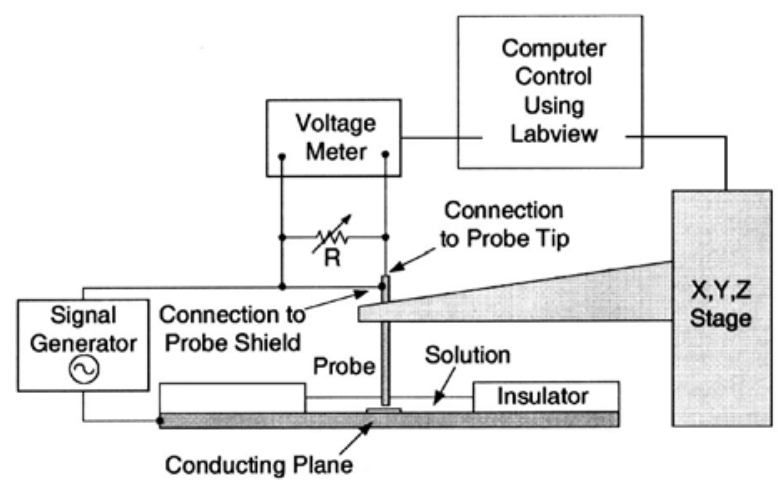

FIG. 1. Diagram of the experimental setup used to test the noncontacts scanning impedance imaging concept.

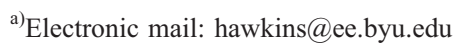




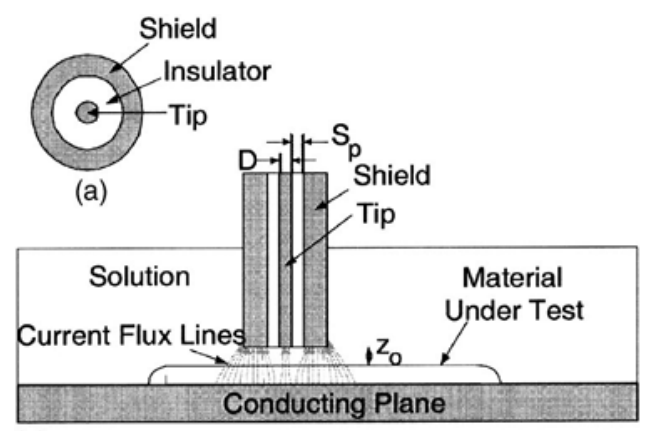

(b)

FIG. 2. Diagram showing a close-up view of the probe used for scanning impedance imaging. The probe consists of an inner conductor (tip) separated by an outer conductor (shield) with an insulator. The shield is used to eliminate current flux originating from areas away from the tip. (a) A top-view cross section of the probe, (b) a side view cross section.

sulator done using a $100 \mu \mathrm{m}$ tip diameter probe with and without shielding. The plot shows the measured rms voltage difference for the probe tip compared to the minimum voltage measured from the tip during the scan (when it is over the Teflon insulator). The unshielded probe was scanned $25 \mu \mathrm{m}$ above the insulator, while the shielded probe was
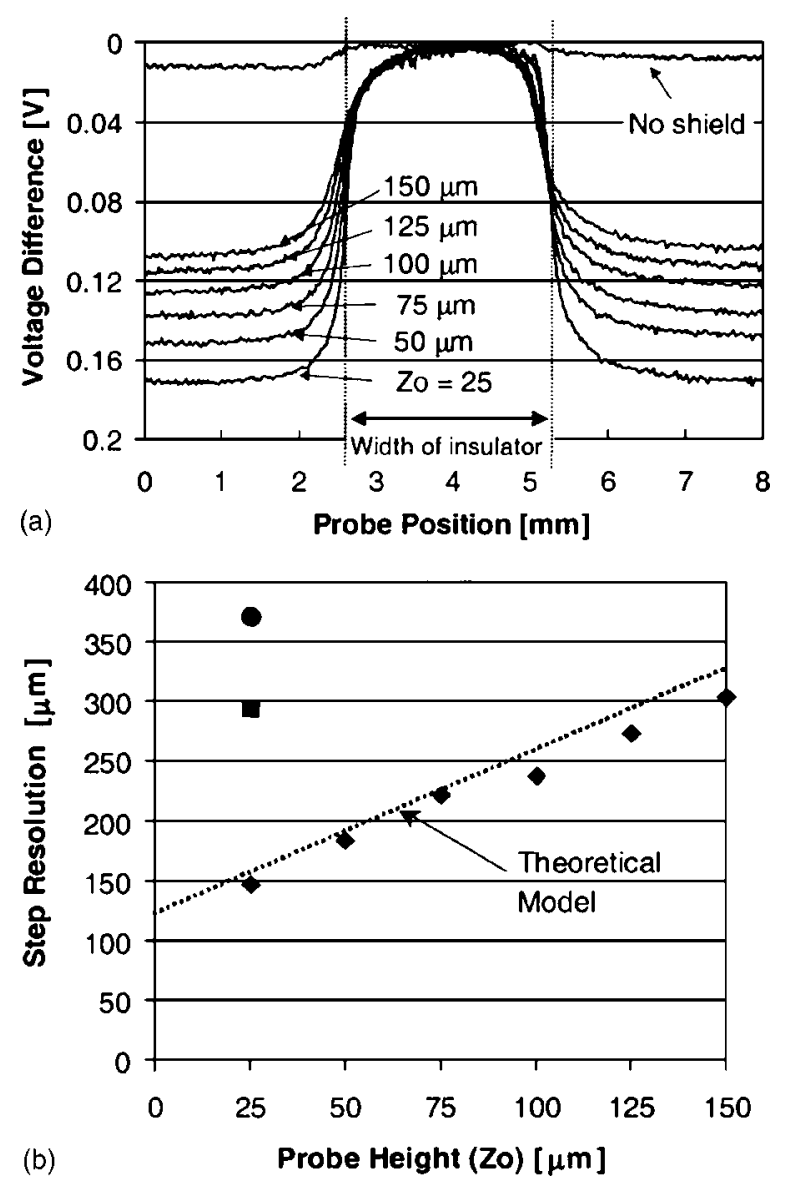

FIG. 3. Measured results of line scans over the insulator. (a) The voltage difference as a function of position for various probe heights for a shielded probe with a $100-\mu \mathrm{m}$-diam probe tip and $75 \mu \mathrm{m}$ shield spacing, and an unshielded probe with a $100-\mu \mathrm{m}$-diam probe tip. (b) The resolution (defined as the distance between the $30 \%$ and $70 \%$ of the maximum voltage difference) as a function of probe height, with (diamonds) a $100 \mu \mathrm{m}$ tip and shield, (square) $250 \mu \mathrm{m}$ tip and shield (180 $\mu \mathrm{m}$ shield spacing), and (circle) $100 \mu \mathrm{m}$ tip with no shield. The theoretical prediction for the resolution is

Fig. 1 was used to produce full two-dimensional impedance
Downloaded 13 Feb 2009 to 128.187.0.164. Redistribution subject to AlP license or copyright; see http://apl.aip.org/apl/copyright.jsp (a)

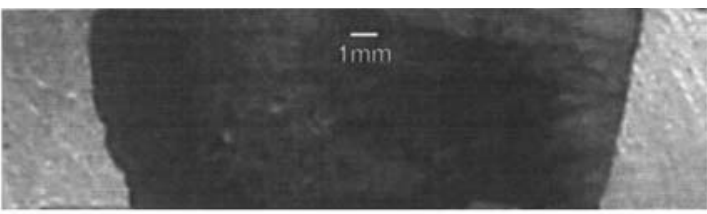

(b)

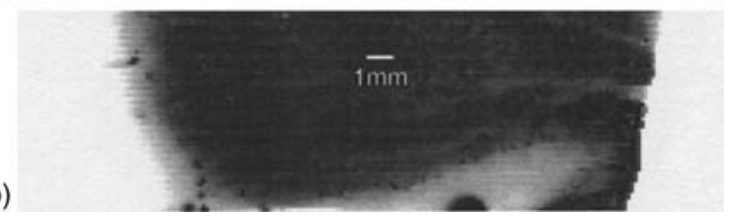

FIG. 4. (a) A digital photo of a flower petal from a geranium sitting directly on a copper plate. (b) An impedance image of the same petal. The scan was produced using a probe with a $100-\mu \mathrm{m}$ diameter probe tip with $75 \mu \mathrm{m}$ shield spacing. The step increment for the image was $125 \mu \mathrm{m}$ in both the $x$ and $y$ directions. The probe was approximately $75 \mu \mathrm{m}$ above the petal's top surface.

scanned at various heights above the insulator as indicated on the plot. The actual width of the insulator is also shown on the graph. The change in impedance as the probe passes over the insulator is indicated by a voltage difference that translates to a change in conductivity between the probe and the conducting plane. As evident from the data, the signalto-noise ratio is much greater in the shielded case and the edges of the insulator are much more apparent. As might be expected, increasing the distance between the probe and the insulator decreases the signal-to-noise ratio and increases the blurring of the abrupt change in impedance that should be present at the edge of the insulator. This blurring can also be effected by the resistivity of the aqueous solution. Lower resistivity solutions would tend to produce lower image resolutions given the same probe geometry and height $Z_{0}$ because the current flux would spread out more rapidly for a given distance above the conducting plane.

Figure 3(b) shows some indication of the resolution possible using SII and various probes. This plot shows the distance between the points with $30 \%$ and $70 \%$ of the maximum voltage difference for line-scans under various conditions. This translates into how effectively the probe can indicate the abrupt change in impedance created by the insulator. In addition to the data from the line-scans found in Fig. 3(a), data for a shielded probe with a $250 \mu \mathrm{m}$ tip are also shown. As can be seen from the figure, the smaller tip provides better resolution of the insulator's edge and is a good indicator that further tip size reduction will produce further resolution improvements.

The data from the line-scans in Fig. 3 were fit to a low frequency, linear model where the current and conductivity are related by a convolution. The convolution uses a blurring function that takes into account the distance $(h)$ between the tip and the ground plane. The one-dimensional step-response implied by this blurring model is $s(x)=\arctan (x / h) / \pi+\frac{1}{2}{ }^{6}$ Figure 3(b) shows a close fit between measured resolution data and the model built from this step response. It should be noted that the height parameter that best fit the data was approximately $1 / 3$ to $1 / 4$ of the true height above the conductor. This is most likely due to the effect of shielding which was not taken into account by the model but it effectively decreases the distance between the probe and the ground plane and decreases the net blurring expected.

In addition to line-scans, the SII system diagramed in Fig. 1 was used to produce full two-dimensional impedance AIP license or copyright; see http://apl.aip.org/apl/copyright.jsp 
maps for several different types of materials. Some of the most dramatic images to emerge were those for organic tissues. Figure 4 shows the results for a geranium flower petal. Figure 4(a) shows the black and white image of the flower petal sitting on a copper plate as taken by a digital camera. Figure 4(b) shows the impedance map of that same petal. The plot shows measured voltage differences indicated by changes in intensity on a grayscale. The pixel size for this image is $125 \times 125 \mu \mathrm{m}^{2}$. Large veins are visible that show up as having different impedance than the rest of the petal. Also visible are areas of lower impedance not necessarily predicted from the digital photo (especially the light areas near the bottom of the petal).

In summary, we have built and demonstrated an imaging process that utilizes impedance measurements through a material. Imaging takes place while samples are surrounded by water in a noncontact configuration. A design for a shielded impedance probe applicable to this process has been outlined and tested. Line-scan data from a high impedance object have been presented and fit to a simple physical model. Results show that probe miniaturization should allow scaling to higher resolutions. Two-dimensional impedance images of objects have also been made including images of biological tissues that show features not readily apparent from standard optical microscopy.

${ }^{1}$ G.J. Saulnier, R.S. Blue, J.C. Newell, D. Isaacson, and P.M. Edic, IEEE Signal Process. Mag. 18, 31 (2001).

${ }^{2}$ A. Malich, T. Fritsch, R. Anderson, T. Boehm, M.G. Freesmeyer, M. Fleck, and W.A. Kaiser, Eur. J. Radiol. 10, 1555 (2000).

${ }^{3}$ V. A. Cherepenin, A.Y. Karpov, A.V. Korjenevsky, V.N. Komienko, Y.S. Kultiasov, M.B. Ochapkin, O.V. Trochanova, and J.D. Meister, IEEE Trans. Med. Imaging 21, 662 (2002).

${ }^{4}$ R. Shao, S. V. Kalinin, and D. A. Bonnell, Appl. Phys. Lett. 82, 1869 (2003).

${ }^{5}$ F. Lacy, M. Kadima-Nzuji, F. J. Malveaux, and E. L. Carter, IEEE Trans. Biomed. Eng. 43, 218 (1996).

${ }^{6}$ J.D. Jackson, Classical Electrodynamics, 2nd ed. (Wiley, New York, 1975), Chap. 2, pp. 54-79. 